\title{
APEM
}

\section{A comparative study of preference dominance-based approaches for selection of industrial robots}

\author{
Chatterjee, P. ${ }^{a,{ }^{*}}$, Mondal, S. ${ }^{b}$, Chakraborty, S. \\ ${ }^{a}$ Department of Mechanical Engineering, MCKV Institute of Engineering, Howrah - 711204, West Bengal, India \\ ${ }^{b}$ Department of Production Engineering, Mallabhum Institute of Technology, Bankura - 722122, West Bengal, India \\ 'Department of Production Engineering, Jadavpur University, Kolkata - 700032, West Bengal, India
}

\section{A B S T R A C T}

In the modern era of highly mechanized technologies, manufacturing organizations are now extensively using different kinds of industrial robots for performing complicated and perilous tasks with superior levels of accuracy. The major role of robotic technology within manufacturing organizations is to amalgamate design, manufacturing and management planning activities into a flexible system for improving production lines with minimum manufacturing cost involvement. However, the pre-implementation, implementation, and post-implementation phases of robotic technologies are the foremost issues associated with the selection and rationalization of robotic investments, which is based on a thorough review and exploration of various alternative robots and their mutually conflicting performance measures. Evaluating alternative robots in the presence of multiple conflicting attributes often makes the selection task very complex. This paper focuses on the application feasibilities of two preference dominance-based multi-attribute decision-making (MADM) approaches, namely evaluation of mixed data (EVAMIX) and extended preference ranking organization method for enrichment evaluation II (EXPROM2) whilst selecting the best alternative robots within given manufacturing environments. Using these two methods, a list of all the feasible alternatives from the best to the worst suitable robot is obtained by taking into account different robot selection attributes. The ranking performances of these methods are also compared with those of the past researchers, using four performance tests.
\end{abstract}

\section{ARTICLE INFO}

Keywords:

Industrial robot selection

Multi-attribute decision-making

EVAMIX

EXPROM2

Performance comparison

*Corresponding author: prasenjit2007@gmail.com (Chatterjee, P.)

Article history:

Received 8 August 2013

Revised 24 February 2014

Accepted 27 February 2014 


\section{References}

[1] Goh, C.H. (1997). Technical note: Analytic hierarchy process for robot selection, Journal of Manufacturing Systems, Vol. 16, No. 5, 381-386, doi: 10.1016/S0278-6125(97)81731-1.

[2] Saen, R.F. (2006). Technologies ranking in the presence of both cardinal and ordinal data, Applied Mathematics and Computation, Vol. 176, No. 2, 476-487, doi: 10.1016/j.amc.2005.09.037.

[3] Bhangale, P.P., Agrawal, V.P., Saha, S.K. (2004). Attribute based specification, comparison and selection of a robot, Mechanism and Machine Theory, Vol. 39, No. 12, 1345-1366, doi: 10.1016/j.mechmachtheory.2004.05.020.

[4] Rao, R.V., Padmanabhan, K.K. (2006). Selection, identification and comparison of industrial robots using digraph and matrix methods, Robotics and Computer-Integrated Manufacturing, Vol. 22, No. 4, 373-383, doi: 10.1016 Lj.rcim.2005.08.003.

[5] Shih, H.-S. (2008). Incremental analysis for MCDM with an application to group TOPSIS, European Journal of Operational Research, Vol. 186, No. 2, 720-734, doi: 10.1016/i.ejor.2007.02.012.

[6] Chatterjee, P., Athawale, V.M., Chakraborty, S. (2010). Selection of industrial robots using compromise ranking and outranking methods, Robotics and Computer-Integrated Manufacturing, Vol. 26, No. 5, 483-489, doi: 10.1016/j.rcim.2010.03.007.

[7] Kumar, R., Garg, R.K. (2010). Optimal selection of robots by using distance based approach method, Robotics and Computer-Integrated Manufacturing, Vol. 26, No. 5, 500-506, doi: 10.1016/j.rcim.2010.03.012.

[8] Athawale, V.M., Chakraborty, S. (2011). A comparative study on the ranking performance of some multi-criteria decision-making methods for industrial robot selection, International Journal of Industrial Engineering Computations, Vol. 2, No. 4, 831-850, doi: 10.5267/j.ijiec.2011.05.002.

[9] Rao, R.V., Patel, B.K., Parnichkun, M. (2011). Industrial robot selection using a novel decision making method considering objective and subjective preferences, Robotics and Autonomous Systems, Vol. 59, No. 6, 367-375, doi: 10.1016/i.robot.2011.01.005.

[10] Koulouriotis, D.E., Ketipi, M.K. (2011). A fuzzy digraph method for robot evaluation and selection, Expert Systems with Applications, Vol. 38, No. 9, 11901-11910, doi: 10.1016/j.eswa.2011.03.082.

[11] Devi, K. (2011). Extension of VIKOR method in intuitionistic fuzzy environment for robot selection, Expert Systems with Applications, Vol. 38, No. 11, 14163-14168, doi: 10.1016/j.eswa.2011.04.227.

[12] Athawale, V.M., Chatterjee, P., Chakraborty, S. (2012). Selection of industrial robots using compromise ranking method, International Journal of Industrial and Systems Engineering, Vol. 11, No. 1/2, 3-15, doi: 10.1504/IJISE. 2012.046651.

[13] İç, Y.T. (2012). An experimental design approach using TOPSIS method for the selection of computer-integrated manufacturing technologies, Robotics and Computer-Integrated Manufacturing, Vol. 28, No. 2, 245-256, doi: 10.1016/j.rcim.2011.09.005.

[14] İç, Y.T., Yurdakul, M., Dengiz, B. (2012). Development of a decision support system for robot selection, Robotics and Computer-Integrated Manufacturing, Vol. 29, No. 4, 142-157, doi: 10.1016/j.rcim.2012.11.008.

[15] Bahadir, M.C., Satoglu, S.I. (2012). A decision support system for robot selection based on axiomatic design principles, In: Proceedings of the 2012 International Conference on Industrial Engineering and Operations Management, Istanbul, Turkey, 674-683.

[16] Datta, S., Sahu, N., Mahapatra, S. (2013). Robot selection based on grey-MULTIMOORA approach, Grey Systems: Theory and Application, Vol. 3, No. 2, 201-232, doi: 10.1108/GS-05-2013-0008.

[17] Liu, H.-C., Ren, M.-L., Wu, J., Lin, Q.-L. (2013). An interval 2-tuple linguistic MCDM method for robot evaluation and selection, International Journal of Production Research, 1-14, doi: 10.1080/00207543.2013.854939.

[18] Ketipi, M.K., Koulouriotis, D.E. (2014). Robot evaluation and selection Part A: an integrated review and annotated taxonomy, International Journal of Advanced Manufacturing Technology, Vol. 71, No. 5-8, 1371-1394, doi: 10.1007/s00170-013-5525-5.

[19] Ketipi, M.K., Koulouriotis, D.E., Karakasis, E.G. (2014). Robot evaluation and selection Part B: a comparative analysis, International Journal of Advanced Manufacturing Technology, Vol. 71, No. 5-8, 1395-1417, doi: 10.1007/s00170-013-5526-4.

[20] Martel, J.M., Matarazzo, B. (2005). Other outranking approaches. In: Figueira, J., Salvatore, G., Ehrgott, M. (Eds.), Multiple criteria decision analysis: state of the art surveys, Springer, New York.

[21] Saaty, T.L. (1990). The analytical hierarchy process, McGraw-Hill, New York.

[22] Zou, Z.-h., Yun, Y., Sun, J.-n., (2006). Entropy method for determination of weight of evaluating indicators in fuzzy synthetic evaluation for water quality assessment, Journal of Environmental Sciences, Vol. 18, No. 5, 1020-1023, doi: 10.1016/S1001-0742(06)60032-6.

[23] Hajkowicz, S., Higgins, A. (2008). A comparison of multiple criteria analysis techniques for water resource management, European Journal of Operational Research, Vol. 184, No. 1, 255-265, doi: 10.1016/i.ejor.2006.10.045.

[24] Chung, E.-S., Lee, K.S. (2009). Identification of spatial ranking of hydrological vulnerability using multi-criteria decision making techniques: case study of Korea, Water Resource Management, Vol. 23, No. 12, 2395-2416, doi: 10.1007/s11269-008-9387-9.

[25] Jeffreys, I. (2004). The use of compensatory and non-compensatory multi-criteria analysis for small-scale forestry, Small-scale Forest Economics, Management and Policy, Vol. 3, No. 1, 99-117.

[26] Diakoulaki, D., Koumoutsos, N. (1991). Cardinal ranking of alternative actions: extension of the PROMETHEE method, European Journal of Operational Research, Vol. 53, No. 3, 337-347, doi: 10.1016/0377-2217(91)90067-6.

[27] Raju, K.S., Kumar, D.N. (1999). Multicriterion decision making in irrigation planning, Agricultural Systems, Vol. 62, No. 2, 117-129, doi: 10.1016/S0308-521X(99)00060-8. 
[28] Doumpos, M., Zopounidis, C. (2004). A multi-criteria classification approach based on pair-wise comparison, European Journal of Operational Research, Vol. 158, No. 2, 378-389, doi: 10.1016/i.ejor.2003.06.011.

[29] Chatterjee, P., Chakraborty, S. (2014). Flexible manufacturing system selection using preference ranking methods: a comparative study, International Journal of Industrial Engineering Computations, Vol. 5, No. 2, 315-338, doi: $10.5267 /$ j.ijiec.2013.10.002.

[30] Rao, R.V. (2007). Decision making in the manufacturing environment using graph theory and fuzzy multi attribute decision making methods, Springer-Verlag, London. 


\title{
Primerjalna študija za izbiro industrijskih robotov, ki temelji na pristopih z izbiro prednostnih zmogljivosti
}

\author{
Chatterjee, P. ${ }^{\mathrm{a},{ }^{*},}$, Mondal, S. ${ }^{b}$, Chakraborty, ${ }^{\mathrm{c}}{ }^{\mathrm{c}}$ \\ ${ }^{a}$ Department of Mechanical Engineering, MCKV Institute of Engineering, Howrah - 711204, West Bengal, India \\ ${ }^{b}$ Department of Production Engineering, Mallabhum Institute of Technology, Bankura - 722122, West Bengal, India \\ ${ }^{c}$ Department of Production Engineering, Jadavpur University, Kolkata - 700032, West Bengal, India
}

\section{POVZETEK}

$\mathrm{V}$ dobi visoko mehaniziranih tehnologij, proizvodno naravnana podjetja čedalje pogosteje uporabljajo različne vrste industrijskih robotov za izvedbo zapletenih in nevarnih nalog, ki zahtevajo vrhunsko raven natančnosti. Glavna naloga robotske tehnologije $\mathrm{v}$ proizvodnih organizacijah je združiti načrtovanje, proizvodnjo in menedžment $v$ prilagodljiv sistem, ki izboljšuje proizvodna postrojenja in minimizira proizvodne stroške. Vendar pa so faze uvajanja robotske tehnologije pred njeno izvedbo, v času izvedbe in po njej, v prvi vrsti povezane $\mathrm{z}$ izbiro in racionalizacijo investicije $\mathrm{v}$ robotizacijo, ki temelji na temeljitem pregledu in raziskavi različnih alternativnih robotov in njihovih medsebojno nasprotujočih si kriterijev zmogljivosti. Vrednotenje različnih robotov z več nasprotujočimi si kriteriji zmogljivosti bistveno otežuje izbor. Članek se osredotoča na dva pristopa, ki omogočata $v$ danem proizvodnem okolju izbiro najboljše alternative robotov. Oba postopka temeljita na prednostnem večparameterskem odločitvenem pristopu (MADM): prvi postopek vrednoti mešane podatke (EVAMIX), drugi pa vključuje razširjeno prednostno rangiranje za obogateno vrednotenje II (EXPROM2). Z uporabo teh dveh postopkov je bil dobljen seznam vseh možnih alternativ, od najbolj primernega robota do najmanj primernega, ob upoštevanju različnih parametrov. Rangirane zmogljivosti s pomočjo opisanih metod smo primerjali tudi s preteklimi izsledki drugih avtorjev, kjer so bili uporabljeni štirje preizkusi zmogljivosti.
\end{abstract}

\section{PODATKI O ČLANKU}

Ključne besede:

Izbira industrijskega robota

Večparametrsko odločanje

EVAMIX

EXPROM2

Primerjava zmogljivosti

*Kontaktna oseba: prasenjit2007@gmail.com (Chatterjee, P.)

Zgodovina članka:

Prejet 8. avgusta 2013

Popravljen 24. februarja 2014

Sprejet 27. februarja 2014 